\title{
Research Paper: Morphometric Analytical Study of External Nose Angulations Using Photographic Technique to Derive Reference Values for Sex and Ethnic Group Differentiation in Forensic Identification
}

\author{
Kishan Ramappa Siddapur ${ }^{*}$, Geetha Kishan Siddapur ${ }^{2}$
}

1. Department of Forensic Medicine, Velammal Medical College Hospital and Research Institute, Tamil Nadu Dr. M.G.R. Medical University, Chennai, India. 2. Department of Ear, Nose \& Throat, Velammal Medical College Hospital and Research Institute, Tamil Nadu Dr. M.G.R. Medical University, Chennai, India.

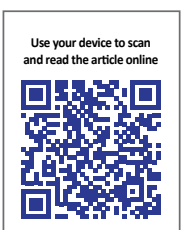

Crtation: Siddapur KR, Siddapur GK. Morphometric Analytical Study of External Nose Angulations Using Photographic Technique to Derive Reference Values for Sex and Ethnic Group Differentiation in Forensic Identification. International Journal of Medical Toxicology \& Forensic Medicine. 2017; 7(3):165-170. https://doi.org/10.18869/nirp.ijmtfm.7.3.165

https://doi.org/10.18869/nirp.ijmtfm.7.3.165

Article info:

Received: 01 Feb. 2017

Accepted: 04 May 2017

\section{Keywords:}

Forensic, Nose, Angle, Identification, Photographic technique

\section{A B S T RACT}

Background: Shape of the nose is unique with regard to ethnicity, race, and sex. Therefore, determining external nose angles provides norms for ethnic and racial differences. Prime objective of the present study was to describe the external nose angulations of Medical students of Tamil Nadu origin (representing South Indian population).

Methods: The present cross-sectional study was done on 118 Medical students from Velammal Medical College Hospital and Research Institute, Tamil Nadu Dr. M.G.R. Medical University, Chennai. Consenting students of Tamil Nadu origin were included. Individuals, other than Tamil Nadu origin, or with noticeable facial disfigurement, or with history of previous facial surgery were excluded. Sample size was calculated using Sample Size Calculator presented as a public service of Creative Research Systems: Survey software, 'The Survey System'.

Results: It was found that the angles were more obtuse in females compared to male counterparts, except for nasofacial and nasomental angles. Based on linear regression graph plotting, low positive correlation was seen to exist between nasal tip and nasolabial angles. However, low negative correlation was found between nasofacial and nasal tip angles. Also, the results were significant with nasofrontal, nasal tip, and nasolabial angles in differentiating gender, i.e., significant difference exists between male and female participants in relation to these angles.

Conclusion: Nasofrontal, nasal tip, and nasolabial angles are useful parameters in differentiating individuals based on gender and ethnicity. These angles differed significantly amongst the gender groups in the present study.

\section{* Corresponding Author:}

Kishan Ramappa Siddapur, MD

Address: Department of Forensic Medicine, Velammal Medical College Hospital and Research Institute, Tamil Nadu Dr. M.G.R. Medical University,

Chennai, India.

Tel: +7 (91) 9500585289

E-mail:kishan_rs@yahoo.co.in 


\section{Introduction}

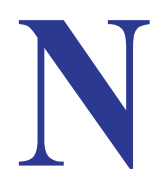

ose shape is one of the parameters in grading looks of an individual. But for a forensic expert, what's more important is the fact that the shape of the nose is unique with respect to ethnicity, race, and sex. Therefore, determining external nose angles also provides norms for ethnic and racial differences [1-3]. Racial and ethnic differences have been the focus of investigations lately $[4,5]$. It is common to find facial parts in mass disasters. Thus, it becomes very important for a forensic expert to ascertain, based on that soft tissue remains, as to which ethnicity and gender the unidentified individual could be. Knowledge of the unique shape of the human nose is also essential for surgeons undertaking esthetic repair and reconstruction of noses $[6,7]$.

External nose angles, as reported by the previous studies are, nasofrontal angle (NFR) [glabella---nasion---pronasale (g---n---prn)], nasofacial angle (NFA) [pronasale---nasion---pogonion (prn---n---pn)], nasal tip angle (NTA) (nasion---pronasale---subnasale (n---prn---sn)], nasolabial angle (NLA) [pronasale---subnasale---labialesuperius (prn---sn---ls)], and nasomental angle (NMA) [nasion- pronasale ---pogonion (n---prn---pn)]. NFR is at a point where forehead ends and the nose starts. It is usually within millimeters away from the upper eyelid skin crease. When the angle is lower to this level, the nose appears relatively small. Normally, this angle ranges between 115 to 135 degrees (relatively greater in females).

NFA has a relation to the nasal septum and the facial plane. In broad noses, NFA is relatively more acute than with narrow noses. Normally it ranges between 30 to 40 degrees. NTA and NLA are complementary to each other. Normally, both angles range from 85 to 105 degrees and females tend to have more obtuse values compared to males. NMA normally ranges from 120 to 130 degrees [8-10]. Prime objective of the present study was to describe the external nose angulations of Medical students (representing South Indian population) from Velammal Medical College Hospital and Research Institute, Madurai, Tamil Nadu. It was also intended to compare results of the present study with that of previous studies reported by various authors.

\section{Materials and Methods}

The present cross-sectional study was conducted from January 2017 to April 2017. The study was approved by Institutional Ethics Committee. Written informed consent was taken from the participants before data collection. A total of 118 (43 males and 75 females) second year Medical students (19-21 years old) from Velammal Medical College Hospital and Research Institute, Madurai, Tamil Nadu, representing South Indian population participated in the study. Consenting students of Tamil Nadu origin were included (inclusion criteria). Individuals, other than Tamil Nadu origin, or with noticeable facial disfigurement and those with history of previous facial surgery were excluded (exclusion criteria). Sample size was calculated using Sample Size Calculator presented as a public service of creative research systems: survey software, 'The Survey System'. Thus, the sample size was 118 (Confidence Level at 95\%, and Confidence Interval of 4) of total 142 students (population).

Participants were positioned in anatomical posture with head positioned in Frankfurt Horizontal plane (FH). FH plane is defined by a line connecting the orbital (the lowest point of the infraorbital margin) and the porion (point at the upper edge of the auditory meatus) or tragion (landmark on the upper edge of the tragus), and can be achieved by judging the relationship of upper and lower edges of the ear to the eye brow level and the ala level, respectively [8].

Photographic technique was used to record data. Lateral view photograph of face of each participant was

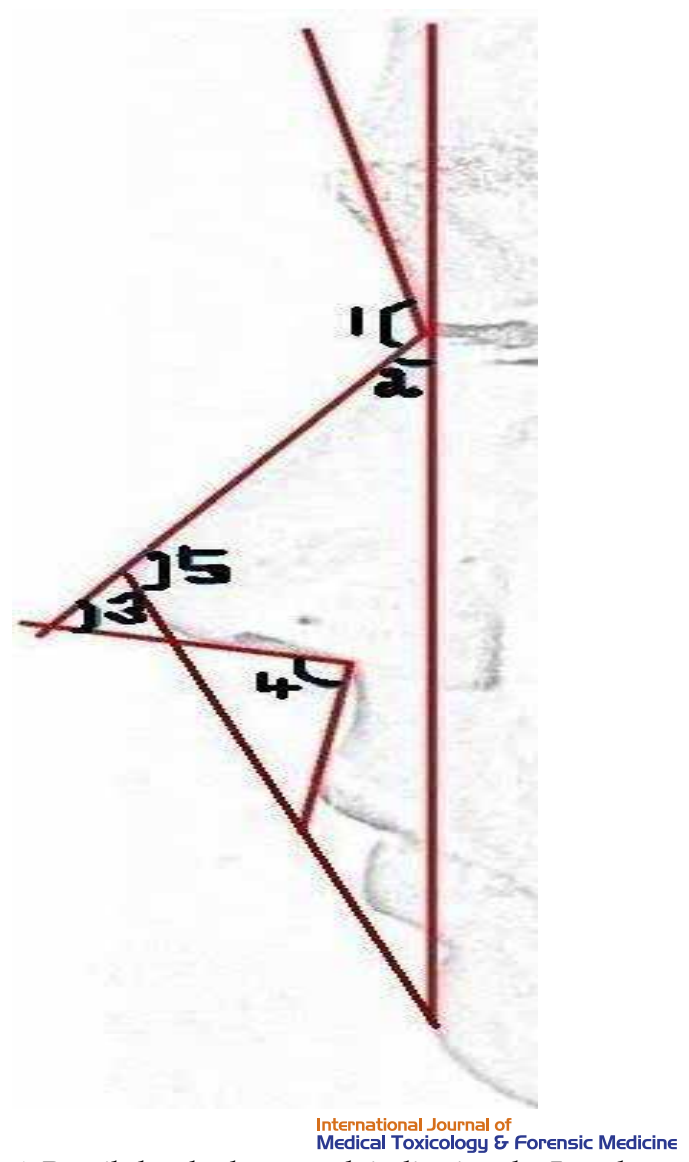

Figure 1. Pencil sketch photograph indicating the 5 angles 
Table 1. Statistical findings of the measured angles

\begin{tabular}{|c|c|c|c|c|c|c|c|c|c|c|}
\hline \multirow{2}{*}{ Variables/n } & \multicolumn{2}{|c|}{ Nasofrontal } & \multicolumn{2}{|c|}{ Nasofacial } & \multicolumn{2}{|c|}{ Nasal Tip } & \multicolumn{2}{|c|}{ Nasolabial } & \multicolumn{2}{|c|}{ Nasomental } \\
\hline & Mean & SD & Mean & SD & Mean & SD & Mean & SD & Mean & SD \\
\hline Males (43) & 126.2 & 8.8 & 35.7 & 3.8 & 84.3 & 4.3 & 88.6 & 9.3 & 127.2 & 4.3 \\
\hline Females (75) & 135.1 & 5.5 & 34.9 & 2.9 & 93.6 & 4.9 & 104 & 8.1 & 126.3 & 4 \\
\hline Total (118) & 131.9 & 8.1 & 35.2 & 3.3 & 90.2 & 6.5 & 98.4 & 11.3 & 126.6 & 4.1 \\
\hline
\end{tabular}

taken. These photographs were later converted to pencil sketch photographs (using Android software). This was done to get a clear outline of the lateral facial profile as in Figure 1, and also, to eliminate the investigator bias (by masking the identity of the participants). Each such photograph was proforma for data collection from each participant. Measurements of the angles were taken using MB-Ruler (Marcus Bader-Ruler) software. Using this software it is possible to accurately measure angles and distances between target points on desktop images.

The analyzed parameters were 1) NFR, 2) NFA, 3) NTA, 4) NLA, and 5) NMA as indicated in Figure 1. The measurements were noted in degrees $\left({ }^{\circ}\right)$. Mean and standard deviations of measured variables were calculated. Statistical analysis was done by the application of unpaired t-test using Graphpad software. Significance level was assessed with $\mathrm{P}$ value less than 0.05 .

\section{Results}

Observed values for all measured angles were tabulated into a Master-Chart. Statistical values namely, mean, Standard Deviation (SD), Standard Error of Mean (SEM), and $95 \%$ confidence interval of the difference in mean of the parameters of Male (M) and Female (F) participants were estimated. Intermediate values in

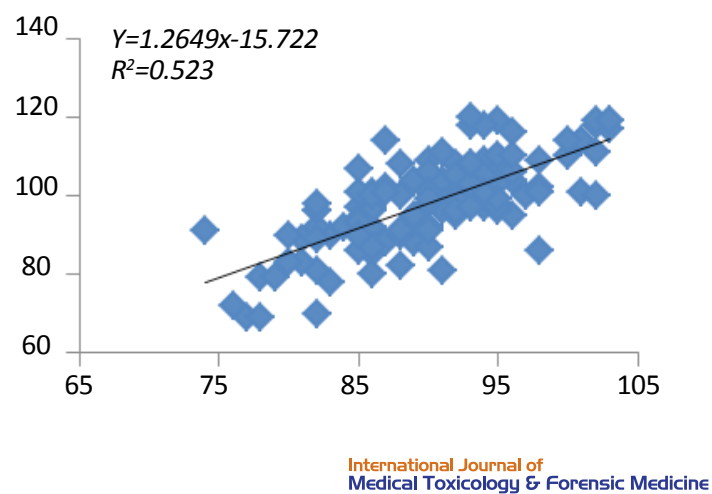

Figure 2. Linear regression graph for NTA (horizontal axis) \& NLA (vertical axis) calculation were t-value, Degrees of Freedom (df), and Standard Error of Difference (SED). Table 1 presents statistical findings of the measured angles. Findings of NFR and NLA in the present study were more interesting due to the fact that male values had higher standard deviation despite being lower (126.2 and 88.6 degrees, respectively) compared to females (135.1 and $104 \mathrm{de}-$ grees, respectively). This means that these angles in females are consistently more obtuse than those of males.

It is also obvious from Table 1 that the angles were more obtuse in females compared to male counterparts, except for NFA and NMA. During the study, authors were quite curious to know if any correlation existed amongst NTA, NFA and NLA as these angles existed in close proximity. Interestingly, from linear regression graph, it was found that low positive correlation did exist between NTA and NLA (Figure 2). However, low negative correlation was found between NFA and NTA, as indicated by Figure 3.

Statistical analysis (Table 2), with the application of unpaired t-test, was done to assess if there was significant difference between male and female nasal angles. The results were significant with NFR, NTA and NLA, i.e., significant difference existed between male and female participants in relation to these angles.

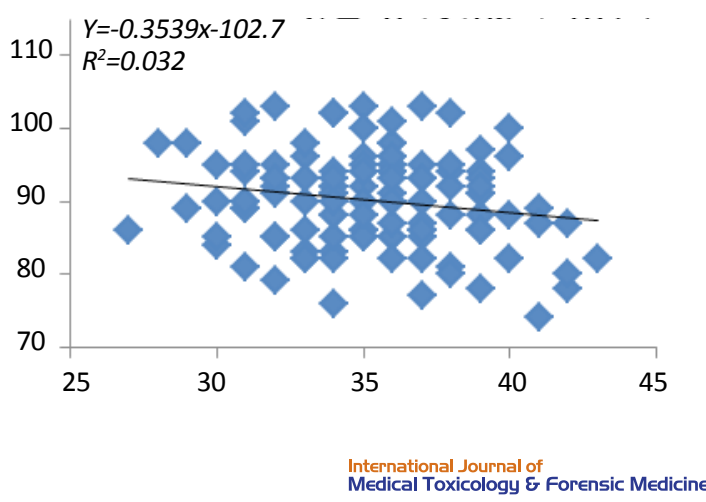

Figure 3. Linear regression graph for NFA (horizontal axis) \& NTA (vertical axis) 
Table 2. Statistical analysis between male and female nasal angles with unpaired t-test

\begin{tabular}{ccccccc}
\hline & $\mathbf{9 5 \%} \mathbf{C l}$ & $\mathbf{t}$ & $\mathbf{d f}$ & $\mathbf{S E D}$ & $\mathbf{P}$ & Statistical Significance \\
\hline Nasofrontal & 6.3 to 11.5 & 6.7477 & 116 & 1.319 & Less than 0.0001 & Significant \\
\hline Nasofacial & 0.45 to 2.02 & 1.2607 & 116 & 0.625 & 0.2099 & Not Significant \\
\hline Nasal tip & 7.56 to 11.09 & 10.441 & 116 & 0.893 & Less than 0.0001 & Significant \\
Nasolabial & 12.1 to 18.6 & 9.3769 & 116 & 1.641 & Less than 0.0001 & Significant \\
\hline Nasomental & 2.45 to 0.67 & 1.1254 & 116 & 0.791 & 0.2627 & Not Significant \\
\hline
\end{tabular}

\section{Discussion}

Similar studies have been done in the past by various authors. But present study is the first of its kind to report on all 5 external nasal angles in a certain population. Table 3 presents reported results by various authors. Uzun and Ozdemir (2014) reported on NFR, NTA, and NLA of the external nose in Turkish population [8]. Interestingly, their study reported acute values for female NTA, which meant that high proportion of Turkish females have pointed noses. Eliakim-Ikechukwu et al. [9] reported results for 2 ethnic groups, Ibo and Yoruba in 2013.

Both ethnic group findings were more or less similar to that of the present study except for the NLA, whose values were notably low in that study for both male and female categories (76.1 and 82.5 degrees respectively for Ibo group, and 77 and 84 degrees, respectively for Yoruba group). The present study reports 88.6 and 104 degrees for NLA of males and females, respectively. Maxillary prominence could be an important reason for acute NLA in Ibo and Yoruba ethnic groups. Extremely Significant difference in NFR between male (132 degrees) and female (100.6 degrees) participants was reported by Anibor and associates [10]. The study was done on Urhobo ethnic group of Nigeria in 2010. Interestingly, this was the only study that reported least obtuse value for NFR in females. Normally it is more obtuse in females. This implies that the females of Urhobo ethnic group have protruded nose and more prominent glabella compared to the male counterparts.

Studies by Raza et al. [11], Kommi et al. [12], Dua et al. [13], and Fitzerald [14] were all done on NLA. Most obtuse NLA (113.6 and 116.2 degrees for males and females, respectively) was reported by Fitzerald (1992). His study was done on white (Caucasian) adults. Nasolabial soft tissue thickness could be an important index factor for NLA variations reported in all these studies. Asian populations supposedly have greater nasolabial soft tissue thickness and therefore have a more acute NLA compared to the white ethnic group, wherein, flat facial contours are more frequent. Minimal variations were observed in relation to NFA and NMA amongst ethnic groups of various studies. However, when it comes to

Table 3. Results reported by various authors

\begin{tabular}{|c|c|c|c|c|c|c|c|c|c|c|c|}
\hline \multirow{2}{*}{\multicolumn{2}{|c|}{$\begin{array}{l}\text { Various } \\
\text { Studies }\end{array}$}} & \multicolumn{2}{|c|}{ NFR } & \multicolumn{2}{|c|}{ NFA } & \multicolumn{2}{|c|}{ NTA } & \multicolumn{2}{|c|}{ NLA } & \multicolumn{2}{|c|}{ NMA } \\
\hline & & $\mathbf{M}$ & $\mathbf{F}$ & $\mathbf{M}$ & $\mathbf{F}$ & $\mathbf{M}$ & $\mathbf{F}$ & $\mathbf{M}$ & $\mathbf{F}$ & M & $\mathbf{F}$ \\
\hline \multicolumn{2}{|c|}{ Present study } & 126.2 & 135.1 & 35.7 & 34.9 & 84.3 & 93.6 & 88.6 & 104 & 127.2 & 126.3 \\
\hline \multicolumn{2}{|c|}{ Uzun et al. [8] } & 123.9 & 133.2 & & & 82.2 & 77.9 & 97.9 & 98.9 & - & - \\
\hline Eliakim-Ikechuk- & Ibo group & 127.1 & 131.7 & 37.8 & 36.3 & - & - & 76.1 & 82.5 & 125.9 & 125.1 \\
\hline wu et al. [9] & Yoruba group & 127.9 & 134.3 & 37.3 & 35.5 & - & - & 77.0 & 84.0 & 125.6 & 126.8 \\
\hline \multicolumn{2}{|c|}{ Anibor et al. [10] } & 132.0 & 100.6 & 39.6 & 37.4 & - & - & - & - & 127.0 & 127.4 \\
\hline \multicolumn{2}{|c|}{ Raza et al. [11] } & - & - & - & - & - & - & 106.4 & 105.2 & - & - \\
\hline \multicolumn{2}{|c|}{ Kommi et al. [12] } & - & - & - & - & - & - & 101.7 & 98 & - & - \\
\hline \multicolumn{2}{|c|}{ Dua et al. [13] } & - & - & - & - & - & - & 96.7 & 95.6 & - & - \\
\hline \multicolumn{2}{|c|}{ Fitzerald [14] } & - & - & - & - & - & - & 113.6 & 116.2 & - & - \\
\hline
\end{tabular}


esthetic repair and reconstruction of noses, both angles are important parameters, and cannot be ignored.

Present study methodology was similar to that adopted by Uzun and associates [8]. However, Eliakim-Ikechukwu et al. [9] and Anibor et al. [10] also adopted similar methodology, except for the fact that they used lateral photograph printouts and then measured the angles on the hard copies. An instrument like 'Goniometer' could have been used to measure the angles; however, the present study methodology (photography technique) is an excellent method of documentation and is fairly easy to use. Studies like Raza et al. [11] and Dua et al. [13], which dealt with NLA only, used cephalometric radiographs (cephalograms) for measuring the angle. In cephalometric radiographs the image is lifelike; however, it is expensive and requires unnecessary exposure of the participants.

\section{Conclusion}

From the present study, it can be concluded that NFR, NTA, and NLA are useful parameters in differentiating individuals based on gender and ethnicity in Forensic identification, as significant differences exist, in relation to these parameters, between the gender and the ethnic groups. Also, maxillary prominence and nasolabial soft tissue thickness contribute to a major extent for variation in these angles amongst the ethnic groups. However, as observed from the present and the previous studies, values for nasofacial and nasomental angles were not significant in differentiating the individuals based on gender and ethnic groups, but as already discussed, they are of immense importance when it comes to esthetic repair and reconstruction of noses, and therefore cannot be ignored.

\section{Acknowledgements}

This research did not receive any specific grant from funding agencies in the public, commercial, or not-forprofit sectors. Authors thank all students who volunteered to participate in the study. We also acknowledge the immense help received from the scholars whose articles are cited and included in the references of this article. The authors are also grateful to authors, editors, and publishers of all those articles, journals, and books from where the literature for this article has been reviewed and discussed. Study was approved by the Institutional Ethics Committee (Ethics Code:VMCIEC/46/2016).

\section{References}

[1] Fedok FG, Burnett MC, Billingsley EM. Small nasal defects. Otolaryngologic Clinics of North America. 2001;34(4):671-94. doi: 10.1016/s0030-6665(05)70013-x.

[2] Uzun A, Akbas H, Bilgic S, Emirzeoglu M, Bostancı O, Sahin $\mathrm{B}$, et al. The average values of the nasal anthropometric measurements in 108 young Turkish males. Auris Nasus Larynx. 2006; 33(1):31-5. doi: 10.1016/j.anl.2005.05.004

[3] Farkas LG. Anthropometry of the head and face in medicine $2^{\text {nd }}$ edition. New York: Raven Press; 1994.

[4] Porter JP. The average African American male face. Archives of Facial Plastic Surgery. 2004; 6(2):78-81. doi: 10.1001/archfaci.6.2.78

[5] Rhee SC, Kang SR, Park HS. Balanced angular profile analysis. Plastic and Reconstructive Surgery. 2004; 114(2):535-44. PMID: 15277828

[6] Echinard C, Dantzer E. Reconstruction of the nose in deep extensive facial burns. Annales de Chirurgie Plastique Esthétique. 1995; 40(3):238-50. PMID: 7574401

[7] Nguyen DD, Turley PK. Changes in the Caucasian male facial profile as depicted in fashion magazines during the twentieth century. American Journal of Orthodontics and Dentofacial Orthopedics. 1998; 114(2):208-17. doi: 10.1053/ od.1998.v114.a86137

[8] Uzun A, Ozdemir F. Morphometric analysis of nasal shapes and angles in young adults. Brazilian Journal of Otorhinolaryngology. 2014; 80(5):397-402. doi: 10.1016/j.bjorl.2014.07.010

[9] Eliakim-Ikechukwu CF, Ekpo AS, Etika M, Ihentuge C, Mesembe OE. Facial aesthetic angles of the ibo and yoruba ethnic groups of Nigeria. IOSR Journal of Pharmacy and Biological Sciences. 2013; 5(5):14-17. doi: 10.9790/3008-0551417

[10] Anibor E, Okumagma MT. Photometric facial analysis of the urhobo ethnic group in Nigeria. Archives of Applied Science Research. 2010; 2(3):28-32.

[11] Raza HA, Rasool G, Ijaz W, Nausheen A. Evaluation of the nasolabial angle of Khyber pakhtunkhwa sample. Pakistan Oral \& Dental Journal. 2014; 34(1):80-82

[12] Kommi PB, Venkatesan R, Keerthi N, Kumar AN, Kumar $\mathrm{S}$, Gopinath V. A cephalometric assessment of ideal nasolabial angle range for south Indian population. Journal of International Oral Health. 2016; 8(2):205-207.

[13] Dua V, Gupta S, Singh C. Evaluation of the nasolabial angle in the Indian population. Contemporary Clinical Dentistry. 2010; 1(2):79-82. doi: 10.4103/0976-237x.68595

[14] Fitzgerald JP, Nanda RS, Currier GF. An evaluation of the nasolabial angle and the relative inclinations of the nose and upper lip. American Journal of Orthodontics and Dentofacial Orthopedics. 1992; 102(4):328-34. doi: 10.1016/08895406(92)70048-f

\section{Conflict of Interest}

The authors declared no conflicts of interest. 
